\title{
Parameters Turning of ADRC based on Neural Network
}

\author{
Kan Hongliang \\ School of Information \& Control Engineering \\ Shenyang Jianzhu University \\ Shenyang, China \\ e-mail: arnold0110@sina.com \\ * Corresponding Author \\ Kan Fenglong \\ School of Information \& Control Engineering \\ Shenyang Jianzhu University \\ Shenyang, China \\ Chen Nan \\ School of Information \& Control Engineering \\ Shenyang Jianzhu University \\ Shenyang, China \\ Ma Qiaoshi \\ School of Information \& Control Engineering \\ Shenyang Jianzhu University \\ Shenyang, China
}

\begin{abstract}
Objective:Though ADRC controller shows strong robust character and adaptability, it still exist a lot of shortages, such as the frequency characteristie, stability... ete. Those were not solved in the theories. And the most diffieulty of ADRC's application in the industry is that the parameters are too more and the parameters'adjusting is diffieulty. The engineers go short of the experience of the parameters' adjusting for the eontroller. To solve the problems that the active disturbance rejection controller has too many parameters and it is very difficult to calculate a set of optimal parameters without determinate turning algorithms.2. Methods: Neural network can get the gradient information of the controlled object, then use gradient descent method to modify nonlinear combined parameters online, so that the ADRC controller has the ability of selflearning, enhanced adaptive ability of ADRC. 3 Results :The simulation results show that, the ADRC controller, which has good dynamic and static features, improves the design efficiency. The feasibility and effectiveness of this method is further verified.
\end{abstract}

Key words-tracking differentiator; gradient information; ADRC controller; parameters turning; self-learning

\section{INTRODUCTION}

ADRC was firstly proposed by Han in [1-2], advantages and disadvantages of classical PID are investigated from its basic principles. A new type controller-Active Disturbances Rejection Controller

\author{
Wang Xin \\ School of Information \& Control Engineering \\ Shenyang Jianzhu University \\ Shenyang, China \\ Zhang Dongwei \\ Information \& Control Engineering Faculty \\ Shenyang Jianzhu University \\ Shenyang, China \\ Qi Ning \\ Library \\ Shenyang Jianzhu University \\ Shenyang, China \\ Wang Bin \\ Northeastern University at Qinhuangdao \\ Qinhuangdao, China
}

(ADRC) with excellent characters is constructed via some links with especial functions, such as Tracking Differentiator (TD), Extended States Observer (ESO) and Nonlinear PID (NPID), which are based on the nonlinear control mechanisms. Furthermore, the new active disturbances rejection control technique is formed. The new controller possesses the following characteristics: the algorithm is simple; the adjustment of the parameters is easier. ADRC is a new non-linear algorithm used in different fields in recent years, it has been proposed and developped for almost two decades, and its applications can be found in lots of literature in recent years. It is a control method that does not depend on the accurate mathematical model of the unknown object. By real-time estimation and compensations of the internal and external disturbances of system, combining with nonlinear control strategy, it can get better static and dynamic performances, strong robustness and adaptability. Since ADRC does not depend on the accurate model of the system, it is very robust against parameter variations, disturbances and noises, not only in some operation areas but also in the whole working area. Many novel design methods [3-10] for the auto-disturbance-rejection controllers (ADRC) were proposed to deal with the difficulty in the parameter regulation of the ADRC. Bring forward ADRC controller based on NN . The kind of method can adaptively adjust 
the parameters of nonlinear error feedbaek control rule NLSEE .

\section{ADRC STRATEGY}

Classical proportional-integral-derivative (PID) is a particular primitive and simplified implementation of the basic principle in error-based feedback control, which focuses on eliminating the control error by using the current, past and future states of the feedback error. There is a question, that if the load changes in a very large range, we can not change the parameters online to meet the system requirements. However, the ADRC method which doesn't depend on system model can estimate and compensate the effects of all the internal and external disturbances in real-time. ADRC not only has the same advantages of fast response and strong robustness as traditional PID control theory, but also gives a new control theory and control method which is widely applied for its excellent system performances.

The ADRC consists of three parts, a nonlinear TD which is used to obtain the ideal transient process of the system, an ESO which estimates all the disturbances by the system output, and then the ADRC compensates the disturbance according to estimated values, a nonlinear state error feedback (NLSEF) which is used to get the control input of the system. The structure of ADRC controller is shown in Figure.1.

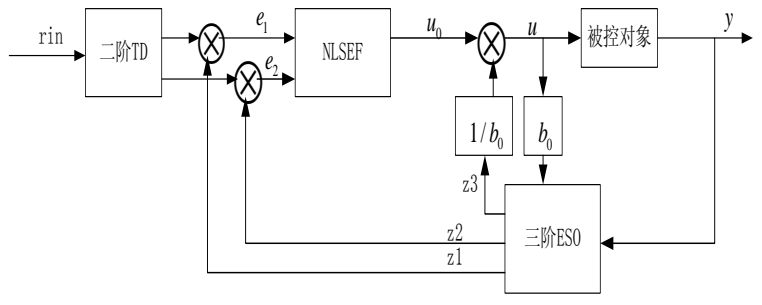

Figure 1. The structure of ADRC system

\section{A. $\quad T D$}

One feasible second-order TD can be designed as

$\left\{\begin{array}{l}x_{1}(k+1)=x_{1}(k)+h x_{2}(k) \\ x_{2}(k+1)=x_{2}(k)+\text { fhan }\left(x_{1}(k), x_{2}(k), r, h_{0}\right)\end{array}\right.$

Where $\mathrm{x} 1$ denotes the control objective, $\mathrm{r}$ is the speed factor which decides tracking speed. The greater value of $r$ is, the faster transition process will be. Here h0 is the filtering factor which makes an effort of filtering. As we know, decreasing the integration step will make a great effect on limiting the noise. When the integration step is fixed, increasing the filtering factor will make the filtering effect better. The function is defined as follows:

$$
f h a n\left(x_{1}(k), x_{2}(k), r, h_{0}\right)=\left\{\begin{array}{l}
-r \cdot \frac{\alpha}{d},|\alpha| \leq d \\
-r \cdot \operatorname{sign}(\alpha),|\alpha|>d
\end{array}\right.
$$

$$
\begin{aligned}
& \left\{\begin{array}{l}
d=r \cdot h_{0} \\
d_{0}=d h_{0} \\
y(k)=x_{1}(k)-y_{0}+h_{0} \cdot x_{2}(k) \\
\alpha_{0}=\sqrt{d^{2}+8 r|y(k)|}
\end{array}\right. \\
& \alpha=\left\{\begin{array}{l}
x_{2}+\frac{y(k)}{h_{0}},|y(k)| \leq d_{0} \\
x_{2}+\frac{\operatorname{sign}[y(k)] \cdot\left(\alpha_{0}-d\right)}{2},|y(k)|>d_{0}
\end{array}\right.
\end{aligned}
$$

The TD is such a nonlinear component which provides transition process for expected input $\mathrm{v}$ and differential trajectory of $\mathrm{x} 1$ and its differential $\mathrm{x} 2$. TD has the ability to track the given input reference signal with fast response and no overshoot.

\section{B. $E S O$}

$\mathrm{f}(\mathrm{x} 1, \mathrm{x} 2, \omega(\mathrm{t}), \mathrm{t})$ generally includes three parts: modeling dynamics, uncertain dynamics (or uncertain accelerations) and disturbance; it is difficult to get the exact model of $f$ $(\mathrm{x} 1, \mathrm{x} 2, \omega(\mathrm{t}), \mathrm{t})$ or its approximation. ESO is used to estimate $\mathrm{f}(\mathrm{x} 1, \mathrm{x} 2, \omega(\mathrm{t}), \mathrm{t})$ in real-time and to make adjustments at each sampling point in a digital controller. Here $f(x 1, x 2, \omega(t), t)$ is considered an extended state for the system, $x 3$ is the uncertain $f(x 1, x 2, \omega(t), t)$, and its differential $a(t)$.

We can use the following nonlinear observer to estimate $\mathrm{x}$ and $\mathrm{f}(\mathrm{x} 1, \mathrm{x} 2, \omega(\mathrm{t}), \mathrm{t})$ :

$$
\begin{aligned}
& \left\{\begin{array}{l}
e(k)=z_{1}(k)-\operatorname{yout}(k) \\
z_{1}(k+1)=z_{1}(k)+h\left(z_{2}(k)-\beta_{01} e(k)\right) \\
z_{2}(k+1)=z_{2}(k)+h\left(z_{3}(k)-\beta_{02} f a l\left(e(k), \alpha_{1}, \delta\right)+b_{0} u(k)\right) \\
z_{3}(k+1)=z_{3}(k)-h \beta_{03} f a l\left(e(k), \alpha_{2}, \delta\right)
\end{array}\right. \\
& \operatorname{fal}(x, \alpha, \delta)=\left\{\begin{array}{l}
|x|^{\alpha} \operatorname{sign}(x),|x|>\delta \\
\frac{x}{\delta^{1-\alpha}},|x| \leq \delta
\end{array}\right.
\end{aligned}
$$

\section{Nonlinear combination}

State error feedback control law generates control signal $u$ for system by using the error between the output of ESO and TD. The errors are combined in nonlinear manners; large errors is corresponding to lower gains, and small errors is corresponding to higher gains.

where e1, e 2 are the output errors. PID, as a control law, employs a linear combination of present, accumulative, and predictive forms of the tracking error and has, for along time, ignored other possible combinations that are potentially much more effective. As an alternative, we propose the following nonlinear functions:

$\left\{\begin{array}{l}e_{1}(k+1)=x_{1}(k+1)-z_{1}(k+1) \\ e_{2}(k+1)=x_{2}(k+1)-z_{2}(k+1) \\ u_{0}(k+1)=\beta_{1}(k) f a l\left(e_{1}(k+1), \alpha_{3}, \delta\right)+\beta_{2}(k) f a l\left(e_{2}(k+1), \alpha_{4}, \delta\right) \\ u(k+1)=u_{0}(k+1)-z_{3}(k+1) / b_{0}\end{array}\right.$

ADRC parameter Self-learning algorithm adopts optimization index: 
$J=\frac{1}{2}(\operatorname{rin}(k)-y(k))^{2}$

error $=r(k)-y(k)$

$\beta 1, \beta 2$ can use gradient descent method to modify nonlinear combined parameters.

$$
\begin{aligned}
& \beta_{1}(k)=\beta_{1}(k-1)-\eta \frac{\partial J}{\partial \beta_{1}} \\
& \beta_{2}(k)=\beta_{2}(k-1)-\eta \frac{\partial J}{\partial \beta_{2}}
\end{aligned}
$$

Using the neural network [3] or TD to recognize Jacobian information $\frac{\partial y}{\partial u}$.

\section{THE EXPERIMENTAL RESULTS}

Simulation example use transfer function of reference[4]:

$$
G(s)=\frac{88.46}{4.11 s^{2}+2.63 s+1}, \text { ADRC parameters: }
$$

step=0.1ms;TD: $r=r_{1}=r_{2}=35 ; h_{0}=h_{1}=h_{2}=0.1 \mathrm{~ms}$; ESO:

$$
\alpha_{1}=0.5 ; \alpha_{2}=0.25 ; \delta=0.1 \mathrm{~ms} ; \beta_{01}=600 ; \beta_{02}=120000 ;
$$

$\beta_{03}=8000000 ; \mathrm{b} 0=41.9 ; \mathrm{NLSEF}: \alpha_{3}=1 ; \alpha_{4}=0.5 ; \beta_{1}=70$;

$$
\beta_{2}=1.0
$$

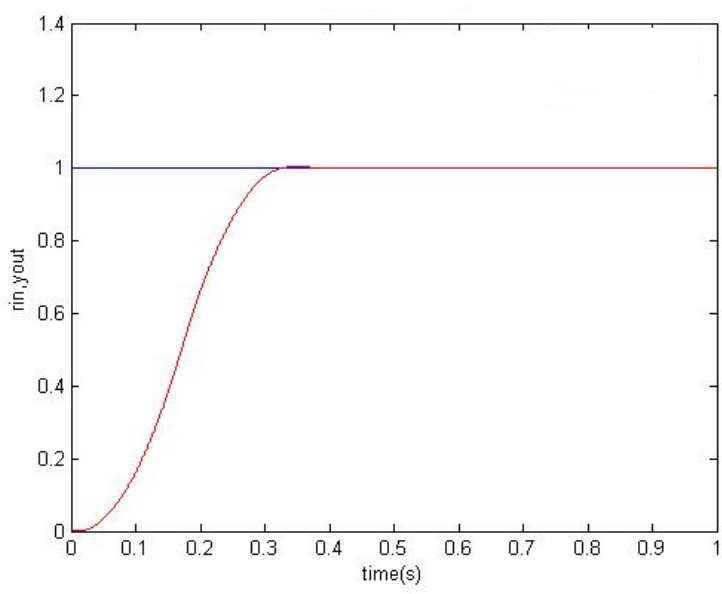

Figure 2. Step response of Servo system

\section{CONCLUSIONS}

Theoretical analysis and simulation results show that the proposed neural network application to extract the gradient information of the object being controlled in ADRC parameter online modification is effective and feasible, improve the quality control system, reduce the design difficulty.

\section{REFERENCES}

[1] HAN Jingqing. Active Disturbance Rejection Control Techniquethe Technique for Estimating and Compensating the Uncertain-ties [M]. Beijing: National Defense Industry Press, 2008.

[2] HAN J Q. From PID to active disturbance rejection control [J]. IEEE Transactions on Industrial Electronics, 2009, 56(3): $900-$ 906.

[3] QIAO Guolin, TONG Chaonan, SUN Yikang. Study on mould level and casting speed coordination control based on ADRC with DRNN optimization [J]. Acta Automatica Sinica, 2007, 33(6): 641 -648$.

[4] HU Yi, WANG Mingang, YANG Yao .Parameters turning of ADRC base on AFSH[J].Command control \& simulation, 2013, 35 (2) :90-92.

[5] Wang Xin,Guo Lili,Ma Lina. Cooperative Spectrum Sensing Algorithm Based on Second User Selection and Random Forest Classification[J].ICIC Express Letters, Part B: Applications,2015, 6(3): 845-850.

[6] LIU Fucai, JIA Yafei, REN Lina. Anti-synchronizing different chaotic systems using active disturbance rejection controller based on the chaos particle swarm optimization algorithm [J]. Acta Physica Sinica, 2013, 62(12): 120509-1 $-120509-8$

[7] LIU Zhaohua, ZHANG Jing, ZHANG Yingiie, et al. ADRC and CMAC combined optimization and control for a class of discretetime uncertain chaotic systems [J]. Acta Physica Sinica, 2011, 60(3):030701-1-030701-9.

[8] Wang Xin, Huang Kuan, Gao Zhijun. The Primary Users Signals Recognition Algorithm in Cognitive Radio Networks via KPCA and Random Forest[J].ICIC Express Letters, 2015, 9(4):1083-1088.

[9] HAN Jingqing, Hou Zengguang.Tracking Differentiator Approaches for Solving Optimization Problems and Finding Roots of Algebraic Equations[J]. Control And Decision, 2000,5:365-367.

[10] LIU Zhaohua, ZHANG Yingjie, ZHANG Jing, et al. A novel binarystate immune particle swarm optimization algorithm [J]. Control Theory \& Applications, 2011, 28(1): $65-72$. 